\title{
The Essential Spectrum of the Linearized 2D Euler Operator is a Vertical Band
}

\author{
Roman Shvidkoy and Yuri Latushkin
}

\begin{abstract}
We prove that the essential spectrum of the operator obtained by linearization about a steady state of the Euler equations governing the motion of inviscid ideal fluid in dimension two is a vertical strip whose width is determined by the maximal Lyapunov exponent of the flow induced by the steady state.
\end{abstract}

\section{Introduction}

In this note we continue the work in $[\mathbf{S L}$, and give a full description of the essential spectrum for the linearized Euler operator $L$ in dimension two. We prove that the essential spectrum of the operator is one solid vertical strip symmetric with respect to the imaginary axis. The width of the strip is determined by the maximal Lyapunov exponent $\Lambda$ for the flow induced by the steady state.

For classical results concerning linearized Euler equations see, e.g., $\mathbf{C}, \mathbf{D R}$, L, Y]. Recent advances concerning the essential spectrum of the linearized Euler operator can be found in FSV, FSV2, FV, FV2, VV, VF. In particular, it was proved in $\mathbf{V}, \mathbf{V F}$ that the essential growth bound for the group generated by $L$ is equal to $\Lambda$. Using this result, it was proved in [LV] that the essential spectral bound for $L$ is equal to $\Lambda$.

We study the linearized Euler operator $L$ in vorticity form,

$$
L w=-\langle u, \nabla\rangle w-\left\langle\operatorname{curl}^{-1} w, \nabla\right\rangle \operatorname{curl} u,
$$

on the Sobolev space $H_{1}^{0}=H_{1}^{0}\left(\mathbb{T}^{2}\right)$ of scalar functions $w$ having zero means $\int w d x=$ 0 on the 2 -torus $\mathbb{T}^{2}=\mathbb{R}^{2} / 2 \pi \mathbb{Z}^{2}$. Here $u=\left(u_{1}, u_{2}\right)^{\top}$ is a steady state (velocity) solution of the Euler equations $\langle u, \nabla\rangle u+\nabla p=0$, div $u=0, p$ is the pressure, $\langle\cdot, \cdot\rangle$ denotes the scalar product, $T-$ transposition, $\operatorname{curl} u=-\partial_{2} u_{1}+\partial_{1} u_{2}$ is the scalar curl of the two-dimensional vector field, and $v=\operatorname{curl}^{-1} w$ denotes the unique solution of $\operatorname{curl} v=w, \operatorname{div} v=0$.

1991 Mathematics Subject Classification. 76,35.

Key words and phrases. Inviscid fluids, Euler equations, essential spectrum.

The second author was partially supported by the Twinning Program of the National Academy of Sciences and National Science Foundation, and by the Research Council and Research Board of the University of Missouri.

(C)0000 (copyright holder) 
Observe, that in the representation $L=-A+T, A w=\langle u, \nabla\rangle w, T w=$ $-\left\langle\operatorname{curl}^{-1} w, \nabla\right\rangle \operatorname{curl} u$, the operator $T$ is compact. The operator $A$ generates the evolution semigroup $e^{t A} w(x)=w \circ \varphi_{t}(x)$, cf. $\mathbf{C L}$. Here $\varphi_{t}: x_{0} \mapsto x\left(t ; x_{0}\right)$ is the flow on $\mathbb{T}^{2}$ generated by the steady state $u$, that is, by the solutions of the equation $\partial_{t} x(t)=u(x(t))$.

Our approach is to describe $\sigma_{a p}(L)$, the approximative point spectrum of $L$, by looking for a weakly null approximative eigenfunction for $A$, that is, for an $\alpha=\lambda+i \xi \in \mathbb{C}$ and for a sequence $\left\{f_{n}\right\} \subset H_{1}^{0}$ such that $\left\|f_{n}\right\|_{H_{1}^{0}}=1, \lim _{n \rightarrow \infty} \|(A-$ a) $f_{n} \|_{H_{1}^{0}}=0$, and $\left\{f_{n}\right\}$ converges to 0 weakly in $H_{1}^{0}$ as $n \rightarrow \infty$. As soon as this sequence is found, $(L+\alpha) f_{n}=-(A-\alpha) f_{n}+T f_{n} \rightarrow 0$ in $H_{1}^{0}$ as $n \rightarrow \infty$, and hence, $-\alpha \in \sigma_{a p}(L)$.

We modify a construction presented in $\mathbf{S L}$, and find weakly null approximative eigenfunctions for $A$ supported in thin and long flow boxes around stable and unstable orbits for the hyperbolic stagnation point $y$ where the maximal Lyapunov exponent $\Lambda$ is attained. To describe our construction, let $u^{\perp}=\left(-u_{2}, u_{1}\right)^{\top}$ and denote by $\left\{\psi_{t}\right\}$ the flow on $\mathbb{T}^{2}$ induced by $u^{\perp}$. Fix $x_{0}=x_{1}$ on the stable orbit (respectively, $x_{0}=x_{2}$ on the unstable orbit) of $y$. Define a bijection $H(t, \tau)=\varphi_{t} \circ \psi_{\tau}\left(x_{0}\right)$ on a strip $\mathcal{S}=\left\{(t, \tau) \in \mathbb{R}^{2}:|t| \leq N\right.$ and $\left.|\tau| \leq s\right\}$, where $s$ is sufficiently small and $N$ is arbitrarily large. Note that under the transformation $H$ the operator $A$ becomes simply $\partial_{t}$. For $\alpha=\lambda+i \xi \in \mathbb{C}$ let

$$
F(t, \tau)=e^{\alpha t} \gamma(t) \beta(\tau), \quad(t, \tau) \in \mathcal{S},
$$

where smooth cut-off functions $\beta$ and $\gamma$ are appropriately chosen such that $\operatorname{supp} \beta \subset$ $(-s, s)$ and $\operatorname{supp} \gamma \subset(-N, N)$. Put $f=F \circ H^{-1}$. Then, by a direct computation, we have:

$$
A f-\left.\alpha f\right|_{H(t, \tau)}=\tilde{F}(t, \tau), \quad \text { where } \tilde{F}(t, \tau):=e^{\alpha t} \gamma^{\prime}(t) \beta(\tau) .
$$

Note that if $s \rightarrow 0$ then $f \rightarrow 0$ weakly. For $\lambda \in(-\Lambda, \Lambda)$ we will choose below a sequence of functions $\gamma=\gamma_{K, M}$ such that if $K, M \rightarrow \infty$ then $\left\|\tilde{F} \circ H^{-1}\right\|_{H_{1}^{0}} / \| F \circ$ $H^{-1} \|_{H_{1}^{0}} \rightarrow 0$. This shows that $\alpha \in \sigma_{a p}(A)$ and $-\alpha \in \sigma_{a p}(L)$.

We use the following notations: For an operator $B$ on $H_{1}^{0}$ we denote by $\sigma(B)$ and $\sigma_{\text {ess }}(B)$ the spectrum and essential (Weyl) spectrum, by $\operatorname{rsp}(B)$ and $\operatorname{rsp} \operatorname{ess}(B)$ the spectral radius and essential spectral radius, by $\omega(B)=t^{-1} \log \operatorname{rsp}\left(e^{t B}\right)$ and $\omega_{\text {ess }}(B)=t^{-1} \log \operatorname{rsp} \operatorname{ess}\left(e^{t B}\right)$ the growth and essential growth bound of the semigroup $\left\{e^{t B}\right\}$, by $s(B)=\sup \{\operatorname{Re} \alpha: \alpha \in \sigma(B)\}$ the spectral bound. For $\alpha=\lambda+i \xi \in$ $\mathbb{C}$ we denote $\|L+\alpha\|_{\bullet}=\inf \left\{\|(L+\alpha) g\|_{H_{1}}:\|g\|_{H_{1}}=1\right\}$. We write $a \lesssim b$ if $a \leq c b$ for a constant $c$ independent of $a$ and $b$.

Acknowledgments. The authors are grateful to Susan Friedlander and Misha Vishik for many discussions.

\section{Results}

Let $\Sigma$ denote the set of all Lyapunov exponents for the differential $\left\{D \varphi_{t}\right\}$, given by the Multiplicative Ergodic Theorem $[\mathbf{O}$, and $\Lambda=\max \{\lambda: \lambda \in \Sigma\}$ denote the maximal exponent:

$$
\Lambda=\lim _{t \rightarrow \infty} \max _{x \in \mathbb{T}^{2}} t^{-1} \log \left\|D \varphi_{t}(x)\right\|
$$


REMARK 2.1. Since $\operatorname{div} u=0$, if $y$ is a hyperbolic stagnation point for $u$, then $\sigma(D u(y))=\{-\lambda, \lambda\}$, where $\lambda>0$ is the Lyapunov exponent for $\left\{D \varphi_{t}\right\}$ at $y$. Also, if $\lambda \in \Sigma \backslash\{0\}$ then there exists a hyperbolic stagnation point $y$ such that $\lambda$ is the Lyapunov exponent for $\left\{D \varphi_{t}\right\}$ at $y$, see [SL Rem. 6].

Let $p(x)=\inf \left\{t>0: \varphi_{t}(x)=x\right\}$ denote the prime period of $x \in \mathbb{T}^{2}$. We set $p(x)=\infty$ if the point $x$ is nonperiodic. We say that the flow $\left\{\varphi_{t}\right\}_{t \in \mathbb{R}}$ has arbitrarily long trajectories if for each $N \in \mathbb{N}$ there is an $x \in \mathbb{T}^{2}$ such that $p(x) \geq N$.

The following facts have been proved in $\mathbf{S L}$ :

(i) if $\lambda \in \Sigma \backslash\{0\}$ then $\lambda+i \mathbb{R} \subset \sigma_{\text {ess }}(L)$;

(ii) $\omega_{\text {ess }}(L)=\omega_{\text {ess }}(-L)=\Lambda$;

(iii) $s(L)=\omega(L)$;

(iv) if $\left\{\varphi_{t}\right\}$ has arbitrarily long trajectories then $i \mathbb{R} \subset \sigma_{\text {ess }}(L)$; if, in addition, $\Lambda=0$ then $i \mathbb{R}=\sigma_{\text {ess }}(L)$.

In this paper we complete the description of $\sigma_{\text {ess }}(L)$ and $\sigma_{\text {ess }}\left(e^{t L}\right), t \in \mathbb{R}$, as follows.

Theorem. If $\Lambda>0$ then $\sigma_{\text {ess }}(L)=\{\alpha \in \mathbb{C}:|\operatorname{Re} \alpha| \leq \Lambda\}$ and $\sigma_{\text {ess }}\left(e^{t L}\right)=\{z \in$ $\left.\mathbb{C}: e^{-|t| \Lambda} \leq|z| \leq e^{|t| \Lambda}\right\}, t \in \mathbb{R}$.

Remark 2.2. All facts listed above and the Theorem hold true if $\sigma_{\text {ess }}(L)$ is replaced by $\sigma(A)$. For higher regularity Sobolev spaces $H_{m}^{0}, m \in \mathbb{Z}$, one can prove similar assertions replacing $\lambda$ by $m \lambda$ and $\Lambda$ by $|m| \Lambda$.

The main step in the proof of the Theorem is the following proposition from SL (see (10) in the proof of Theorems 1 and 2 there); for completness we sketch its proof in Appendix.

Lemma 2.3. If $\alpha=\lambda+i \xi, \lambda, \xi \in \mathbb{R}, x_{0} \in \mathbb{T}^{2}, N<p\left(x_{0}\right) / 2$, and $\gamma \in$ $H_{1}([-N, N])$ is a real valued cut-off function with $\operatorname{supp} \gamma \subset(-N, N)$, then

$$
\|L+\alpha\|_{\bullet}^{2} \leq \frac{\int_{\mathbb{R}}\left|u \circ \varphi_{t}\left(x_{0}\right)\right|^{2} /\left|u\left(x_{0}\right)\right|^{2} e^{2 \lambda t}\left|\gamma^{\prime}(t)\right|^{2} d t}{\int_{\mathbb{R}}\left|u \circ \varphi_{t}\left(x_{0}\right)\right|^{2} /\left|u\left(x_{0}\right)\right|^{2} e^{2 \lambda t}|\gamma(t)|^{2} d t} .
$$

Proof of Theorem. We prove that $(-\Lambda, \Lambda) \subset \sigma_{a p}(L)$. The rest follows from the Spectral Inclusion Theorem $\exp t \sigma(L) \subset \sigma\left(e^{t L}\right)$ and fact (ii) above.

Using Remark 2.1 pick the hyperbolic stagnation point $y$ such that $\sigma(D u(y))=$ $\{-\Lambda, \Lambda\}$. Fix $\lambda \in(0, \Lambda)$ and consider any point $x_{1}, u\left(x_{1}\right) \neq 0$, that belongs to the orbit attracted to $y$ such that $y=\lim _{t \rightarrow \infty} \varphi_{t}\left(x_{1}\right)$. Respectively, fix $\lambda \in(-\Lambda, 0)$ and consider any point $x_{2}, u\left(x_{2}\right) \neq 0$, that belongs to the orbit repelled from $y$ such that $y=\lim _{t \rightarrow-\infty} \varphi_{t}\left(x_{2}\right)$.

Lemma 2.4. There exists $\epsilon=\epsilon(\lambda)>0$ such that

$$
\begin{gathered}
\sup \left\{e^{\epsilon t} e^{\lambda t}\left|u \circ \varphi_{t}\left(x_{1}\right)\right|: t \geq 0\right\}<\infty ; \\
\sup \left\{e^{-\epsilon t} e^{-\lambda t}\left|u \circ \varphi_{t}\left(x_{2}\right)\right|: t \leq 0\right\}<\infty .
\end{gathered}
$$

The proof of the lemma is given in Appendix.

Fix $K>0, M>0$. We will construct a sequence of cut-off functions $\gamma=$ $\gamma_{K, M} \in H_{1}([-N, N]), N>K+2 M$, such that for all $\xi \in \mathbb{R}$ the right hand side of (2.1) tends to zero as $K, M \rightarrow \infty$. By Lemma 2.3 this implies $\alpha=\lambda+i \xi \in \sigma_{a p}(-L)$ which proves the theorem. 
For $\lambda \in(0, \Lambda)$ the function $\gamma$ is defined as follows: If $t \leq 0$ then $\gamma(t)=0$; if $0<t \leq K$, then $\gamma(t)=t / K$; if $K<t \leq K+M$ then $\gamma(t)=\exp (-(t-K) / M)$; if $K+M<t \leq K+2 M$ then $\gamma(t)=-e^{-1}(t-K) / M+2 e^{-1}$; if $t>K+2 M$ then $\gamma(t)=0$. Denote $x_{0}=\varphi_{-K}\left(x_{1}\right)$, and observe that the right-hand side of (2.1) can be estimated from above by the following expression:

$$
\frac{\left(\frac{1}{K^{2}} \int_{0}^{K}+\frac{1}{e^{2} M^{2}} \int_{K+M}^{K+2 M}\right)\left|u \circ \varphi_{t}\left(x_{0}\right)\right|^{2} e^{2 \lambda t} d t+\frac{1}{M^{2}} \int_{K}^{K+M} e^{-\frac{2(t-K)}{M}}\left|u \circ \varphi_{t}\left(x_{0}\right)\right|^{2} e^{2 \lambda t} d t}{\int_{K}^{K+M} e^{-\frac{2(t-K)}{M}}\left|u \circ \varphi_{t}\left(x_{0}\right)\right|^{2} e^{2 \lambda t} d t} .
$$

By (2.2), $e^{\lambda t}\left|u \circ \varphi_{t}\left(x_{0}\right)\right| \lesssim e^{-\epsilon t}$ uniformly for $t \geq 0$. Therefore, we can pass to the limit as $M \rightarrow \infty$ :

$$
\begin{aligned}
\|L+\alpha\|_{\bullet}^{2} \leq & \frac{K^{-2} \int_{0}^{K}\left|u \circ \varphi_{t-K}\left(x_{1}\right)\right|^{2} e^{2 \lambda t} d t}{\int_{K}^{\infty}\left|u \circ \varphi_{t-K}\left(x_{1}\right)\right|^{2} e^{2 \lambda t} d t} \\
& =\frac{K^{-2} e^{2 \lambda K} \int_{-K}^{0}\left|u \circ \varphi_{t}\left(x_{1}\right)\right|^{2} e^{2 \lambda t} d t}{e^{2 \lambda K} \int_{0}^{\infty}\left|u \circ \varphi_{t}\left(x_{1}\right)\right|^{2} e^{2 \lambda t} d t} \\
& \leq \frac{\|u\|_{\infty}}{K^{2}} \frac{\int_{-\infty}^{\infty} e^{2 \lambda t} d t}{\int_{0}^{\infty}\left|u \circ \varphi_{t}\left(x_{1}\right)\right|^{2} e^{2 \lambda t} d t}
\end{aligned}
$$

Note that the improper integrals converge due to (2.2) and $\lambda>0$. Letting $K \rightarrow \infty$, we have $-\alpha \in \sigma_{a p}(L)$.

For $\lambda \in(-\Lambda, 0)$ the function $\gamma$ is defined as follows. If $t \geq 0$ then $\gamma(t)=0$, if $-K \leq t<0$ then $\gamma(t)=-t / K$, if $-(K+M) \leq t<-K$ then $\gamma(t)=\exp ((t+K) / M)$; if $-(K+2 M) \leq t<-(K+M)$ then $\gamma(t)=e^{-1}(t+K) / M+2 e^{-1}$; and if $t<$ $-(K+2 M)$ then $\gamma(t)=0$. Denote $x_{0}=\varphi_{K}\left(x_{2}\right)$. Then the right-hand side of (2.1) can be estimated from above by the following expression:

$$
\begin{gathered}
\int_{-\infty}^{0}\left|u \circ \varphi_{t}\left(x_{0}\right)\right|^{2} e^{2 \lambda t}\left|\gamma^{\prime}(t)\right|^{2} d t \\
\int_{-\infty}^{0}\left|u \circ \varphi_{t}\left(x_{0}\right)\right|^{2} e^{2 \lambda t}|\gamma(t)|^{2} d t \\
\frac{\left(\frac{1}{e^{2} M^{2}} \int_{-(K+2 M)}^{-(K+M)}+\frac{1}{K^{2}} \int_{-K}^{0}\right)\left|u \circ \varphi_{t}\left(x_{0}\right)\right|^{2} e^{2 \lambda t} d t+\frac{1}{M^{2}} \int_{-(K+M)}^{-K} e^{\frac{2(t+K)}{M}}\left|u \circ \varphi_{t}\left(x_{0}\right)\right|^{2} e^{2 \lambda t} d t}{\int_{-(K+M)}^{-K} e^{\frac{2(t+K)}{M}}\left|u \circ \varphi_{t}\left(x_{0}\right)\right|^{2} e^{2 \lambda t} d t} .
\end{gathered}
$$

By (2.3), $e^{\lambda t}\left|u \circ \varphi_{t}\left(x_{0}\right)\right| \lesssim e^{\epsilon t}$ uniformly for $t \leq 0$. Similarly to the case $\lambda \in(0, \Lambda)$, we conclude that $-\alpha \in \sigma_{a p}(L)$. 


\section{Appendix}

Proof of Lemma 2.4. Choose $v_{1,2} \in \mathbb{R}^{2}$ such that $\left|v_{1}\right|=\left|v_{2}\right|=1$, and $D u(y) v_{1}=-\Lambda v_{1}, D u(y) v_{2}=\Lambda v_{2}$. Then $D \varphi_{t}(y)=e^{t D u(y)}$ and

$$
\lim _{t \rightarrow+\infty} \frac{u \circ \varphi_{t}\left(x_{1}\right)}{\left|u \circ \varphi_{t}\left(x_{1}\right)\right|}=v_{1}, \quad \lim _{t \rightarrow-\infty} \frac{u \circ \varphi_{t}\left(x_{2}\right)}{\left|u \circ \varphi_{t}\left(x_{2}\right)\right|}=v_{2}
$$

imply:

$$
\begin{aligned}
\lim _{t \rightarrow+\infty} \log \left|D \varphi\left(\varphi_{t}\left(x_{1}\right)\right) \frac{u \circ \varphi_{t}\left(x_{1}\right)}{\left|u \circ \varphi_{t}\left(x_{1}\right)\right|}\right| & =-\Lambda, \\
\lim _{t \rightarrow-\infty} \log \left|D \varphi_{-1}\left(\varphi_{t}\left(x_{2}\right)\right) \frac{u \circ \varphi_{t}\left(x_{2}\right)}{\left|u \circ \varphi_{t}\left(x_{2}\right)\right|}\right| & =\Lambda .
\end{aligned}
$$

Note that $u \circ \varphi_{t}(x)=D \varphi_{t}(x) u(x)$ for all $t \in \mathbb{R}$ and $x \in \mathbb{T}^{2}$. Since $\left\|D \varphi_{t}(x)\right\|$ is bounded, it suffices to prove the lemma for $t=k \in \mathbb{Z}$. If $y_{k}=\varphi_{k}\left(x_{1}\right), k=$ $0,1,2, \ldots$, then

$$
\begin{gathered}
\left|u\left(y_{k}\right)\right|=\left|D \varphi\left(y_{k-1}\right) \frac{u\left(y_{k-1}\right)}{\left|u\left(y_{k-1}\right)\right|}\right| \cdot\left|D \varphi\left(y_{k-2}\right) \frac{u\left(y_{k-2}\right)}{\left|u\left(y_{k-2}\right)\right|}\right| \cdot \ldots \\
\cdot\left|D \varphi\left(y_{1}\right) \frac{u\left(y_{1}\right)}{\left|u\left(y_{1}\right)\right|}\right| \cdot\left|D \varphi\left(y_{0}\right) \frac{u\left(y_{0}\right)}{\left|u\left(y_{0}\right)\right|}\right| \cdot\left|u\left(y_{0}\right)\right| .
\end{gathered}
$$

Now (2.4) implies (2.2) with $\epsilon<(\Lambda-\lambda) / 2$. If $y_{k}=\varphi_{k}\left(x_{2}\right), k=0,-1,-2, \ldots$, then

$$
\begin{aligned}
\left|u\left(y_{k}\right)\right| & =\left|D \varphi_{k}\left(x_{2}\right) u\left(x_{2}\right)\right| \\
& =\left|D \varphi_{-1}\left(y_{k+1}\right) \frac{u\left(y_{k+1}\right)}{\left|u\left(y_{k+1}\right)\right|}\right| \cdot\left|D \varphi_{-1}\left(y_{k+2}\right) \frac{u\left(y_{k+2}\right)}{\left|u\left(y_{k+2}\right)\right|}\right| \cdot \ldots \\
& \cdot\left|D \varphi_{-1}\left(y_{0}\right) \frac{u\left(y_{0}\right)}{\left|u\left(y_{0}\right)\right|}\right| \cdot\left|u\left(y_{0}\right)\right| .
\end{aligned}
$$

Now (2.4) implies (2.3) with $\epsilon<(\lambda+\Lambda) / 2$.

Proof of Lemma [2.3. For $H$ defined in the Introduction, we have:

$$
D H(t, \tau)=D \varphi_{t}\left(\psi_{\tau} x_{0}\right)\left[u: \frac{u^{\perp}}{|u|^{2}}\right] \circ \psi_{\tau}\left(x_{0}\right)
$$

and $\operatorname{det} D H=1$. For $F$ and $\tilde{F}$, defined in (1.1) and (1.2), we have:

$$
\nabla F=\left[\begin{array}{c}
\alpha F+e^{\alpha t} \gamma^{\prime}(t) \beta(\tau) \\
e^{\alpha t} \gamma(t) \beta^{\prime}(\tau)
\end{array}\right] ; \quad \nabla \tilde{F}=\left[\begin{array}{c}
\alpha \tilde{F}+e^{\alpha t} \gamma^{\prime \prime}(t) \beta(\tau) \\
e^{\alpha t} \gamma^{\prime}(t) \beta^{\prime}(\tau)
\end{array}\right] .
$$

Choose $\gamma \in H_{1}([-N, N])$ and $\beta(\tau)=(s-|\tau|) \mathbb{I}_{[-s, s]}$, where $\mathbb{I}$ is the characteristic function. If $s \rightarrow 0$ then $|\partial F / \partial t|^{2} /(2 s) \rightarrow 0$ and $|\partial \tilde{F} / \partial t|^{2} /(2 s) \rightarrow 0$ in $L^{2}$. Also, $|\partial F / \partial \tau|^{2} /(2 s) \rightarrow e^{2 \lambda t}|\gamma(t)|^{2} \delta_{0}(\tau)$ and $|\partial \tilde{F} / \partial \tau|^{2} /(2 s) \rightarrow e^{2 \lambda t}\left|\gamma^{\prime}(t)\right|^{2} \delta_{0}(\tau)$ where $\delta_{0}(\cdot)$ is the Dirac $\delta$-function. The measure of the support of $f$ tends to zero as $s \rightarrow 0$, and $f /\|f\|_{H_{1}} \rightarrow 0$ weakly. Since $L=-A+T$, where $T$ is a compact operator, there is a subsequence $s_{j} \rightarrow 0$ such that $\left\|T\left(f /\|f\|_{H_{1}}\right)\right\| \rightarrow 0$. Note that

$$
\left[D H^{-1}(H(t, \tau))\right]^{\top}=\left[D \varphi_{t}\left(\psi_{\tau} x_{0}\right)\right]^{-1 \top}\left[\frac{u}{|u|^{2}}: u^{\perp}\right] \circ \psi_{\tau}\left(x_{0}\right) .
$$


Passing to the $(t, \tau)$ coordinates and letting $j \rightarrow \infty$ we have:

$$
\begin{aligned}
\|L+\alpha\|_{\bullet}^{2} \lesssim & \|A f-\alpha f\|_{H_{1}}^{2} /\|f\|_{H_{1}}^{2}+\left\|T\left(f /\|f\|_{H_{1}}\right)\right\|_{H_{1}}^{2} \\
= & \frac{\left(2 s_{j}\right)^{-1} \int_{\mathcal{S}}\left|D H^{-1 \top} \nabla \tilde{F}\right|^{2} d \tau d t}{\left(2 s_{j}\right)^{-1} \int_{\mathcal{S}}\left|D H^{-1 \top} \nabla F\right|^{2} d \tau d t}+\left\|T\left(f /\|f\|_{H_{1}}\right)\right\|_{H_{1}}^{2} \\
& \rightarrow \frac{\int_{\mathbb{R}}\left|\left[D \varphi_{t}\left(x_{0}\right)\right]^{-1 \top} u^{\perp}\left(x_{0}\right)\right|^{2} e^{2 \lambda t}\left|\gamma^{\prime}(t)\right|^{2} d t}{\int_{\mathbb{R}}\left|\left[D \varphi_{t}\left(x_{0}\right)\right]^{-1 \top} u^{\perp}\left(x_{0}\right)\right|^{2} e^{2 \lambda t}\left(\left.\gamma(t)\right|^{2} d t\right.} .
\end{aligned}
$$

Since $\left[D \varphi_{t}\left(x_{1}\right)\right]^{-1 \top} u^{\perp}\left(x_{0}\right)=u^{\perp} \circ \varphi_{t}\left(x_{0}\right)$, this proves the lemma. To make $f$ meanzero, define another $\bar{f}$ in the same way around the same orbit and disjoint from $f$, varying its support we can obtain $f-\bar{f} \in H_{1}^{0}$.

\section{References}

[C] S. Chandrasekhar, Hydrodynamic and hydromagnetic stability, Clarendon Press, Oxford, 1961.

[CL] C. Chicone and Y. Latushkin, Evolution Semigroups in Dynamical Systems and Differential Equations, Math. Surv. Monogr. 70, AMS, Providence, 1999.

[DR] P. G. Drazin and W. H. Reid, Hydrodynamic stability. Cambridge, 1981.

[FSV] S. Friedlander, W. Strauss, and M. Vishik, Nonlinear instability in an ideal fluid, Ann. Inst. H. Poincaré, Anal. non linéaire 14, 187-209 (1997).

[FSV2] S. Friedlander, W. Strauss, and M. Vishik, Robustness of instability for the twodimensional Euler equations, SIAM J. Math. Anal. 30 (1999), 1343-1354.

[FV] S. Friedlander and M. Vishik, Dynamo theory, vorticity generation, and exponential stretching, Chaos, 1 (1991), 198-205.

[FV2] S. Friedlander and M. Vishik, Instability criteria for steady flows of a perfect fluid, Chaos 2(3) (1992), 455-460.

[LV] Y. Latushkin and M. Vishik, Linear stability in an ideal incompressible fluid, Commun. Math. Phys., to appear.

[L] C. C. Lin, The theory of hydrodynamic stability, Cambridge, 1955.

[O] V. Oseledets, A multiplicative ergodic theorem. Lyapunov characteristic numbers for dynamical systems, Trans. Moscow Math. Soc. 19 (1968), 197-231.

[SL] R. Shvidkoy and Y. Latushkin, Essential spectrum of the linearized $2 D$ Euler equation and Lyapunov-Oseledets exponents. Preprint, 2002.

[V] M. M. Vishik, Spectrum of small oscillations of an ideal fluid and Lyapunov exponents, J. Math. Pures et Appl. 75 (1996), 531-558.

[VF] M. Vishik and S. Friedlander, Dynamo theory methods for hydrodynamic stability, J. Math. Pures Appl. 72 (1993), 145-180.

[Y] Y. Yudovich, The linearization method in hydrodynamical stability theory, Transl. Math. Monogr. 74, AMS, Providence, 1989.

Department of Mathematics, University of Missouri, Columbia, MO 65211

E-mail address: shvidkoy@math.missouri.edu, yuri@math.missouri.edu 